\title{
ACUTE EFFECT OF RESISTED SPRINTING UPON REGULAR SPRINT PERFORMANCE
}

\author{
Roland van den Tillaar ${ }^{1}$, Amaro Teixeira ${ }^{2}$, Daniel Marinho ${ }^{2,3}$ \\ ${ }^{1}$ Department of Sport Sciences and Physical Education, Nord University, Levanger, \\ Norway \\ ${ }^{2}$ Department of Sports Sciences, University of Beira Interior, Covilhã, Portugal \\ ${ }^{3}$ Research Centre for Sport, Health and Human Development, Vila Real, Portugal
}

\begin{abstract}
The aim of this study was to investigate the acute effect of resisted sprinting upon running sprint performance. Thirty male athletes from track and field (age: $21.2 \pm 2.9$ y rs, body mass: $69.8 \pm 9.8 \mathrm{~kg}$, height: $1.75 \pm 0.08 \mathrm{~m}$ ) performed two different test sessions (one day of $7 \times 60 \mathrm{~m}$ runs alternating between regular and resisted sprinting and the other day $7 \times 60 \mathrm{~m}$ of regular sprints) with $5 \mathrm{~min}$ between each run. Sled towing individually weighted to $10 \%$ of each participant's body mass was used as resistance for the resisted sprints. It was found that training with or without resistance had the same effect; there is no acute effect of resisted sprinting upon sprint performance after using resisted runs. It was concluded that resisted sprinting does not have any acute positive effect upon regular sprints of $60 \mathrm{~m}$, but only a fatiguing effect.
\end{abstract}

Keywords: sled running; strength training; sprint training; kinematics; postactivation potentiation

\section{INTRODUCTION}

Sprint training has been essential for success in many sports, including soccer, team handball and of course in athletics. Based on the principles of force overload and specificity [24], there are several ways to increase sprint velocity after an intervention period $[9,29]$. Some examples of specific overload training using force are uphill running, parachute training [15], sprint elastic-towing [6], sled towing $[4,13]$ and running with a loaded, weighted 
vest [11]. The objective of this overload is to elicit a greater neural activation and to increase the recruitment of fast twitch muscle fibres [23].

Resisted sprint running is a type of overload training usually used to improve maximal velocity and acceleration in regular running performance [14]. Sports like rugby, American football, soccer and track and field athletics are examples of sports that use this type of training regularly $[9,10$, $14,23]$. However, intervention studies that used this type of training over a six to nine week period showed discrepant results. Harrison and Bourke [9] found improvement only on the initial $5 \mathrm{~m}$ of $30 \mathrm{~m}$ sprints after six weeks of training with a sled, while Zafeiridis et al. [29] found increased performance on $10 \mathrm{~m}$ and $20 \mathrm{~m}$ sprints after resisted sprint training. However, Kristensen et al. [12] found a decrease in $20 \mathrm{~m}$ sprint performance after six weeks of resisted sprint training. Some other studies [4, 20,23] have also shown increases in sprint performance after a resisted sprint performance, but these improvements were the same as for training groups that conducted a non-resisted sprint program. This indicates that an increased workload (number of sprints) is enough to elicit a greater neural activation, regardless of whether resisted sprints are included [10]. Discrepancy in findings may be caused by the different resistance and training protocols used. However, previous studies $[1,9,29]$ have examined the load-speed relationships and recommended the use of approximately $13 \%$ of body mass or less than $10 \%$ decrease in velocity.

Often these resisted sprints were alternated with normal runs during training or in competition as a warm up protocol to enhance sprint performance straight afterwards $[17,25]$. It is thought that this would stimulate mechanisms like post activation potentiation (PAP), greater neural activation and increased recruitment of fast-twitch muscle fibres [23]. PAP specifically refers to the increase in acute muscle force output as an outcome of contractile history $[8,18,21]$ following a high intensity stimulus like resisted sprints [22]. Generally, this PAP effect appears as a sequence of performance decrement due to acute fatigue followed by enhanced performance [26]. The maximal effect of this PAP phenomenon occurs for approximately 5-10 min only [3]. Thus, this suggests that by including resisted sprints in a warm-up protocol regular sprint performance afterwards could be enhanced. In addition, athletes who warm up or alternate with resisted sprints (sled towing) usually have a subjective perception that they can run faster in a regular sprint due to now experiencing less weight [26]. Van den Tillaar and Von Heimburg [25] found that alternating resisted sprints with regular sprints had a positive effect in $\mathrm{m}$ sprints in handball players. However, this effect was only visible after one resisted sprint. The authors reasoned that handball 
players were not used to this kind of training and therefore several resisted sprints were more fatiguing than the possible positive PAP effect. In athletics resisted sprints are regularly used in training. However, to the best of our knowledge no study has investigated the acute effect of resisted sprinting upon regular sprinting performance in track and field athletes.

Therefore, the purpose of this study was to examine the acute effect of resisted sprinting upon the regular $60 \mathrm{~m}$ sprinting performance of track and field athletes. It was hypothesized that resisted sprint training would lead to running improvements due to mechanisms like PAP, greater neural activation and/or increased recruitment of fast-twitch muscle fibres [23] that could occur after resisted sprint runs.

\section{MATERIALS AND METHODS}

\section{Subjects}

Thirty male athletes (age: $21.2 \pm 2.9$ yrs, body mass: $69.8 \pm 9.8 \mathrm{~kg}$, height: $1.75 \pm 0.08 \mathrm{~m}$ ) from a local track and field club performing on national level in their age category participated in this study. All subjects had regular sprint running experience, but not with sled tow running. Written informed consent was obtained from all participants and parents (if they were under 18 years old), prior to testing and the study was approved by the local committee for medical research ethics and complied with the ethical standards of the Helsinki Declaration and the current ethical standards in sports and exercise research.

\section{Procedures}

To investigate the acute effect of resisted sprints on regular $60 \mathrm{~m}$ sprint performance, a repeated-measures with cross over design was used. This meant that the order of testing with and without resistance was randomized and counter-balanced. The subjects performed two test sessions in which in one session they had to perform seven maximal $60 \mathrm{~m}$ sprints, while on the other test day they sprint seven times alternating with regular and resisted $60 \mathrm{~m}$ sprints. Alternating between the regular and resisted runs was used to investigate how many resisted runs could initiate a PAP effect or when fatigue occurs. In addition, $60 \mathrm{~m}$ runs were chosen to investigate if resisted runs would have an effect on the acceleration and/or maximal velocity phase in sprints. Since it was a cross over design on each test day half of the athletes performed alternating regular and sled running sprints and the other half conducted regular $60 \mathrm{~m}$ sprints only. The second day the protocols were swapped. 
Each participant made themselves available for testing on two non-consecutive days with a break of at least 48 hours or easy training between the testing days (April-May). Before data collection, the age, body mass and height of all participants were recorded and the weight for their sled was calculated for each subject ( $10 \%$ of their body mass) since earlier studies [2, $3,14]$ recommended this weight for enhancing sprint performance after a training period. In the present study, the acute effect of this weight on the sprint performance was investigated to understand if the training effect is an acute effect or an adaptation over time. The sled was attached to a rope that was attached to a harness worn around the shoulders of the participant.

Each participant performed two test sessions of sprints; on one day they performed $7 \times 60 \mathrm{~m}$ sprints alternating between regular and resisted sprint running and on the other day they performed $7 \times 60 \mathrm{~m}$ of regular sprints. $7 \times 60 \mathrm{~m}$ were used in the test sessions, since in their regular training the participants also use this number of maximal $60 \mathrm{~m}$ sprints. In addition, seven sprints alternating between regular and resisted sprints were used to investigate if eventual enhancement occurs after one two or three resisted sprint or that fatigue is influencing the regular $60 \mathrm{~m}$ sprints. A cross over design was used to avoid a learning effect, with the result that on each day half of the athletes performed alternating regular and sled running sprints and the other half conducted regular $60 \mathrm{~m}$ sprints only. $60 \mathrm{~m}$ sprints were used to investigate the effect of the resisted sprints upon both acceleration and maximal running velocity.

All participants performed an identical warm-up routine, which consisted of 10-12 minutes jogging, dynamic exercises for the whole body, followed by three short sprints of increasing intensity. The total time of warm-up lasted approximately $20 \mathrm{~min}$. A standing start with feet in split position one meter behind the first pair of photocells [12] was performed for each run and participants were given five minutes of rest between each run. Approximately $5 \mathrm{~min}$ of rest was chosen since this would give possible maximal PAP effect [3]. Approximately 10 subjects were involved in each testing procedure, which implicated that every $20 \mathrm{~s}$ a maximum sprint was performed by one of the participants. Testing was conducted on an outdoor $400 \mathrm{~m}$ Mondo track (Mondo Spa, Gallo, Italy). In order to reduce the variability of testing, the time of day, the shoes worn, and the testing equipment were standardized for the two tests. The testing sessions were always at the same time of day and on the same days of the week (between 18:00 and 20:00 on a Tuesday or Thursday). Times were recorded at 10,20, 40 and $60 \mathrm{~m}$ using a photocell system (Microgate, Polifemo; Bolzano, Italy). In addition, two video cameras (Casio, Exilim Ex-F1; Montrose, CA, USA) were used: 
one to record the entire run and the other to record the middle of the run, gathering the total number of strides, stride length and stride rate frequency at around $30 \mathrm{~m}$ (part of top velocity during $60 \mathrm{~m}$ ) for each subject for each run (Figure 1). The total number of strides was calculated from the camera that recorded the whole run from start to finish together with the stride length and rate of the whole $60 \mathrm{~m}$ runs. In addition, average stride rate and stride length of three complete cycles from the viewings of the camera that recorded a field of view at 26-31 m of the $60 \mathrm{~m}$ (Figure 1) to investigate these parameters at top speed and to compare them with the parameters measured over the whole runs.

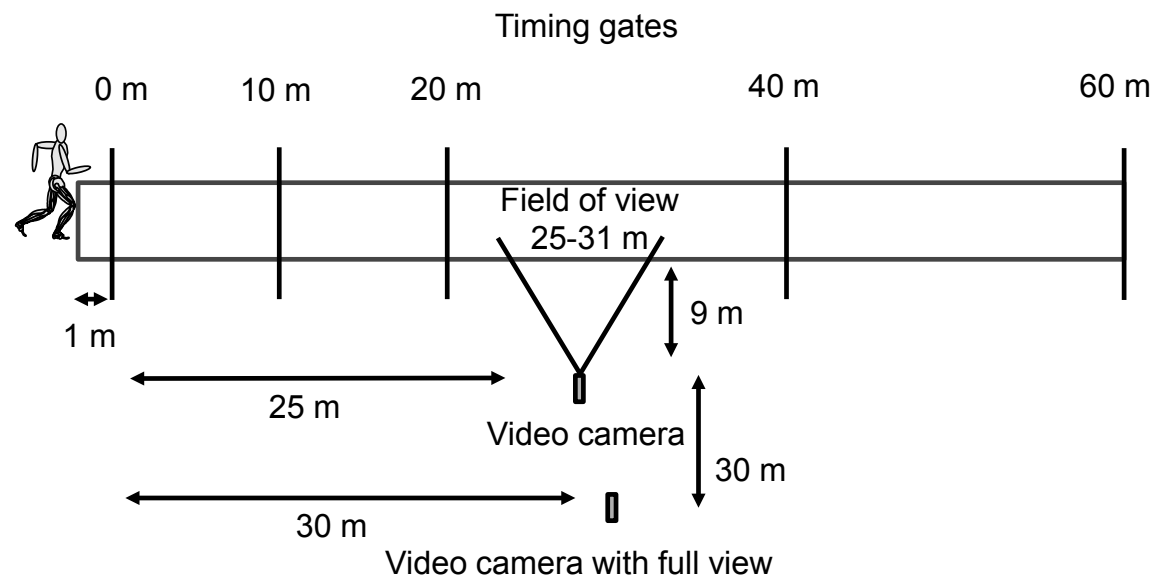

Figure 1. The experimental set up used during data collection.

\section{Statistical analyses}

To assess effects of resisted sprints on the regular $60 \mathrm{~m}$ sprint times, a repeated analysis of variance (ANOVA) 2 (condition: regular sprinting vs. regular and resisted sprints) $\times 4$ (sprint attempt: 1, 3, 5 and 7; regular $60 \mathrm{~m}$ sprints) was used. When significant differences in sprinting times were found a one-way ANOVA was also conducted to locate eventual changes (stride length, rate and number of steps at $60 \mathrm{~m}$ ) for each condition. In addition, also a $1 \times 3$ (sprint attempt: 2,4 , and 6 ) repeated analysis of variance (ANOVA) for the resisted sprint protocol was used to compare the sprint times and kinematics over the three resisted sprint runs. Post hoc comparisons with Holm-Bonferroni corrections were conducted to locate differences. All results are presented as mean $\pm \mathrm{SD}$. When sphericity assumptions were violated, Greenhouse-Geisser adjustments of the p-values were reported. The criterion level for significance was set at $\mathrm{p}<0.05$. Effect size 
was evaluated with $\eta^{2}$ (partial eta squared) where $0.01<\eta^{2}<0.06$ constitutes a small effect, $0.06<\eta^{2}<0.14$ constitutes a medium effect and $\eta^{2}>0.14$ constitutes a large effect [5]. Statistical analysis was performed in SPSS, version 21.0 (SPSS, Inc., Chicago, IL). To test the reliability of the protocol and variability of the day 13 subjects performed the test three times during the testing period and the times on each day were used to calculate the Intraclass Correlation Coefficient (ICC) using Cronbach's alpha.

\section{RESULTS}

The reliability was very high (ICC $=0.988$ ) with no influence due to the day of testing $(\mathrm{F}=0.115 ; \mathrm{p}=0.741)$. The average regular $60 \mathrm{~m}$ sprint times were $8.12 \pm 0.56 \mathrm{~s}$ in both conditions (regular sprint condition and regular and resisted sprint condition). The average sprint time with the sled towing was $8.50 \pm 0.56 \mathrm{~s}$ ( $4.7 \%$ difference). The sprint times did not change significantly during the session $\left(\mathrm{F}=2.31 ; \mathrm{p}=0.117 ; \eta^{2}=0.07\right.$; Figure 2$)$ and no significant differences in running times between the conditions were found $(\mathrm{F}=0.079$; $p=0.781 ; \eta^{2}=0.003$; Figure 2). However, post hoc comparison showed that the running times decreased for the regular condition from sprints 1 to 2 and increased again from 2 to 3 , while under the resisted sprint condition the times increased from sprint 5 onwards (when compared with sprint 3 ). The resisted sprint times increased significantly for each attempt $(\mathrm{F}=14.6$; $\mathrm{p}<0.001 ; \eta^{2}=0.33$; Figure 2).

Also, no significant changes in the regular $10 \mathrm{~m}, 20 \mathrm{~m}$ and $40 \mathrm{~m}$ sprint times (sprint 1, 3, 5 and 7 for each condition) were found ( $\mathrm{F} \leq 1.2 ; \mathrm{p} \geq 0.303$; $\eta^{2} \geq 0.031$; Figure 3 ) and between conditions ( $F \leq 1.1 ; p \geq 0.295 ; \eta^{2} \geq 0.018$; Figure 3). However, after performing a one-way ANOVA on each condition post hoc comparison showed that the running times at 10, 20 and $40 \mathrm{~m}$ increased for the resisted sprint condition the times increased between sprint 3 and 5 and 7 (only 20 and $40 \mathrm{~m}$ times). Under the regular condition the sprint times at $20 \mathrm{~m}$ increased from sprint 2 to 6 and for the $40 \mathrm{~m}$ from sprint 2 to 6 and 7, while 20 and $40 \mathrm{~m}$ times with the sled increased significantly from sprint 4 to 6 (Figure 3 ).

When comparing the interval times between $10-20 \mathrm{~m}, 20-40 \mathrm{~m}$ and $40-60 \mathrm{~m}$, no significant effect of sprint runs $\left(\mathrm{F} \leq 2.5 ; \mathrm{p} \geq 0.082 ; \eta^{2} \geq 0.06\right)$ or condition ( $\mathrm{F} \leq 2.3 ; \mathrm{p} \geq 0.138 ; \eta^{2} \geq 0.003$ ) was found (Figure 4). However, a significant effect during the sled towing sprints at the 20-40 m and 40-60 $m$ intervals were found ( $\mathrm{F} \geq 10.3 ; \mathrm{p} \leq 0.001 ; \eta^{2} \geq 0.22$ ). Post hoc comparison showed that the running times increased during the sled towing sprints 
(sprint 2 to 4 and sprint 4 to 6 ) at the different intervals (Figure 4). Furthermore, interval time at 20-40 and 40-60 m increased from regular sprint 3 to 5 for the resisted sprint condition, while for interval 20-40 time under the regular condition first decreased from sprint 1 to 2 and increased significantly again with sprint 7 (Figure 4).

No significant changes in the total number of steps taken during the seven $60 \mathrm{~m}$ sprints were found $\left(\mathrm{F}=1.57 ; \mathrm{p}=0.156 ; \eta^{2}=0.052\right.$; Figure $\left.5 \mathrm{~A}\right)$, while significant changes in the total number of steps during the seven $60 \mathrm{~m}$ sprints were found when alternating between regular and resisted sprints $\left(\mathrm{F}=16.1 ; \mathrm{p}<0.001 ; \eta^{2}=0.357 ;\right.$ Figure $\left.5 \mathrm{~A}\right)$. Average stride length ( 3.55 vs. 3.80 ) and rate (2.02 vs. 2.08 ) were significantly longer and higher when measured at around $30 \mathrm{~m}$ than during the whole runs in the regular $60 \mathrm{~m}$ runs in both condition $\left(F \geq 94 ; p<0.001 ; \eta^{2}=0.93\right)$ with no significant interaction effect $\left(\mathrm{F} \leq 1.1 ; \mathrm{p} \geq 0.36 ; \eta^{2} \leq 0.01\right)$. Therefore, the stride length and rate around $30 \mathrm{~m}$ were shown in the figures. No significant differences in step length and rate in the regular runs $(1,3,5$ and 7$)$ between the two conditions were found $\left(\mathrm{F} \leq 0.39 ; \mathrm{p} \geq 0.54 ; \eta^{2} \leq 0.013\right)$ and also no interaction effect $(\mathrm{F} \leq 0.62 ; \mathrm{p} \geq 0.46$; $\left.\eta^{2} \leq 0.02\right)$.

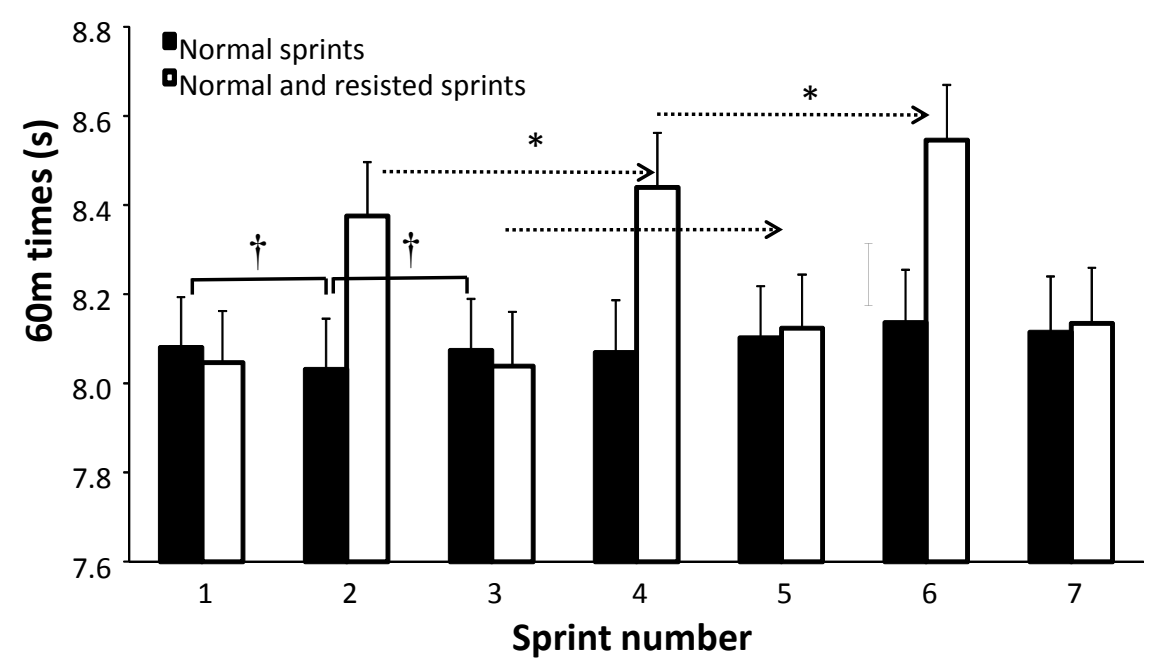

Figure 2. Mean $( \pm S D$ ) sprint times of the seven $60 \mathrm{~m}$ sprints that consisted of either seven regular sprints or three sprints with a sled (sprints 2, 4 and 6) and four sprints without a sled (sprints 1, 3, 5 and 7).

* indicates a significant difference $(p<0.05)$ beween the two sprint times and all those to the right of it (dashed lines for the resisted runs).

t indicates a significant difference $(p<0.05)$ beween these two sprint times. 


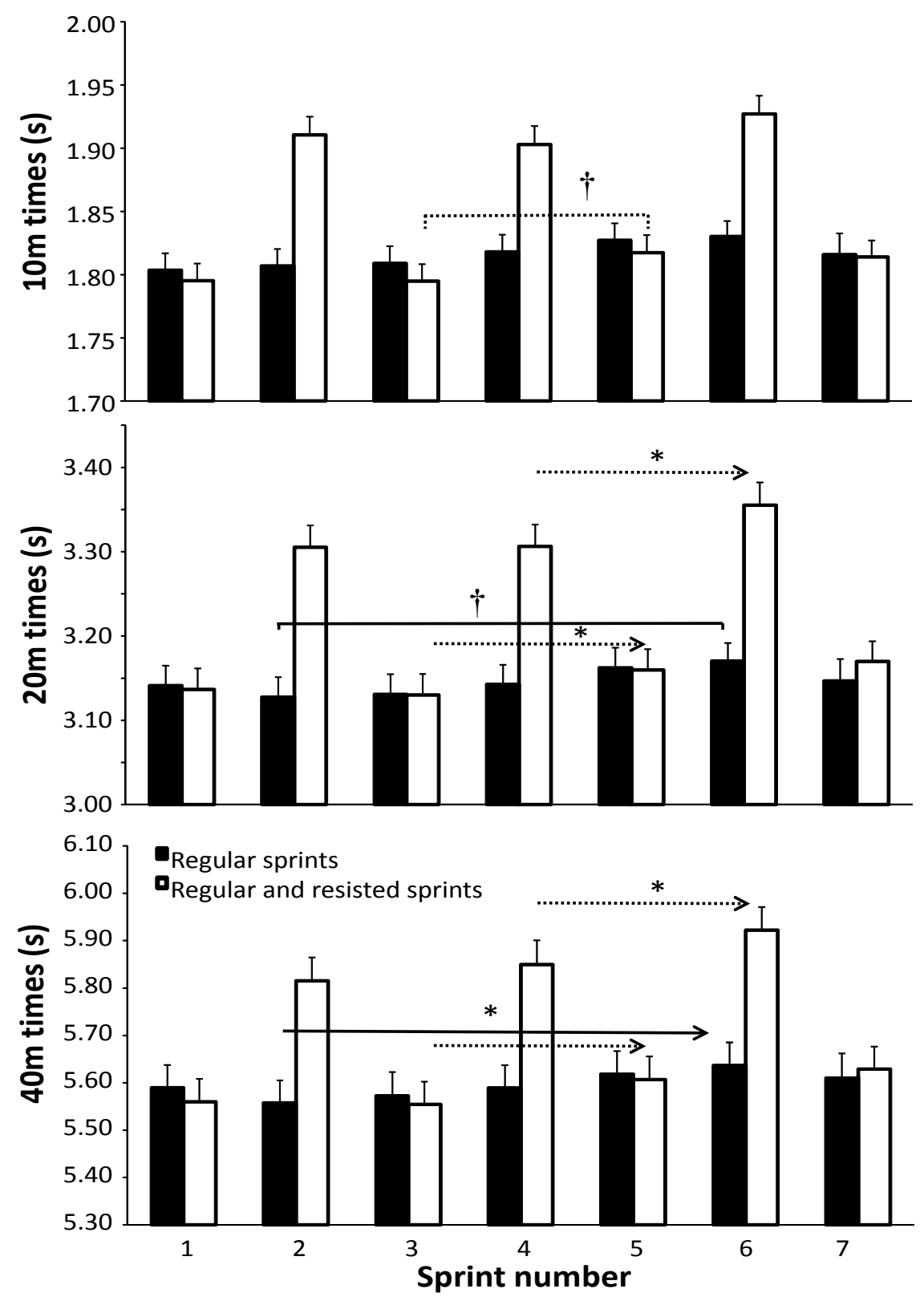

Figure 3. Mean $( \pm S D)$ sprint times at 10,20 and $40 \mathrm{~m}$ in the seven $60 \mathrm{~m}$ sprints that consisted of either seven regular sprints or three sprints with a sled (sprints 2, 4 and 6) and four sprints without a sled (sprints 1, 3, 5 and 7).

* indicates a significant difference $(p<0.05)$ beween these two sprint times and all those to the right of it (dashed lines for the resisted runs, black lines for the regular sprints). t indicates a significant difference $(p<0.05)$ beween these two sprint times. 

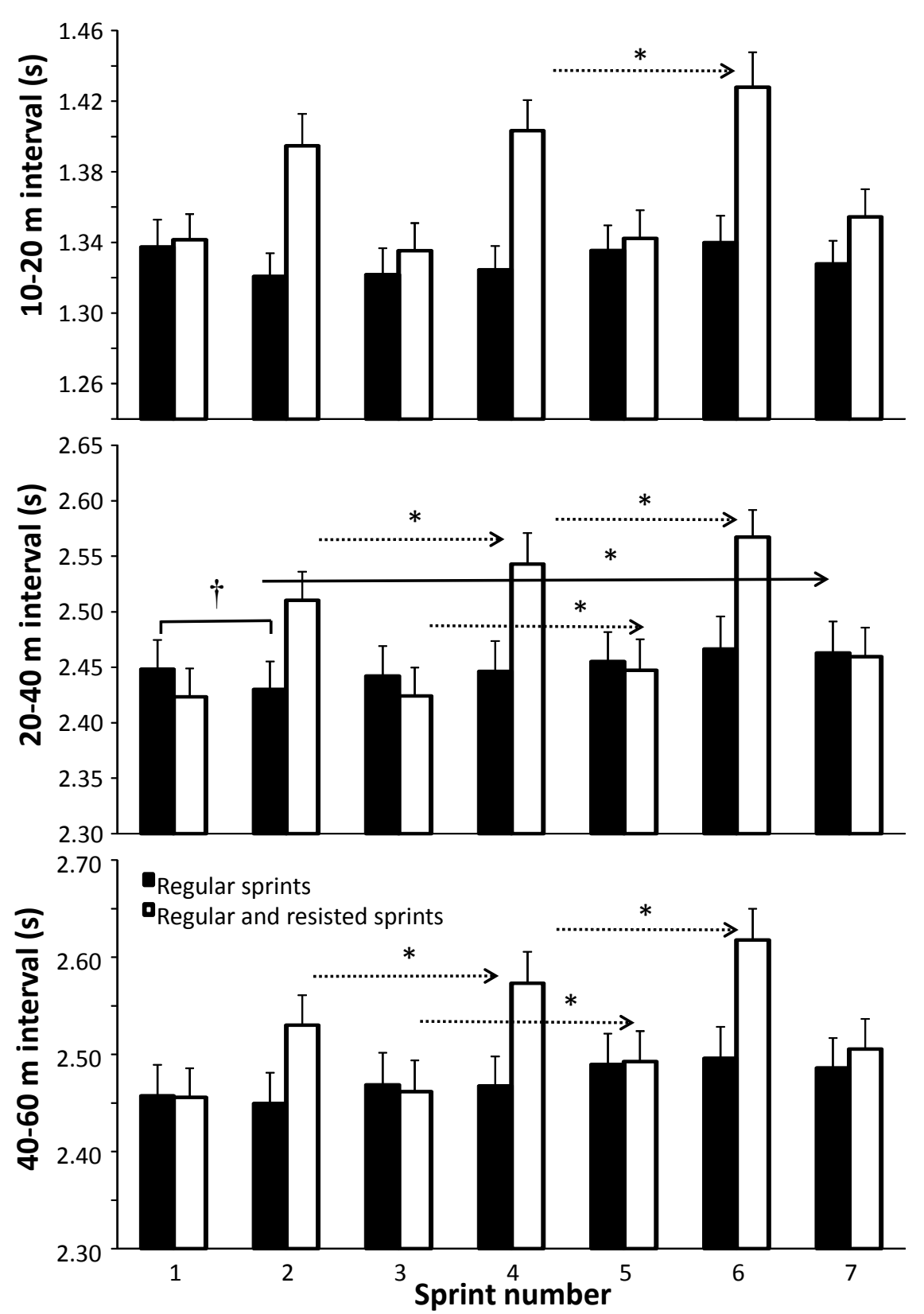

Figure 4. Mean $( \pm S D$ ) sprint times for the intervals $10-20 \mathrm{~m}, 20-40 \mathrm{~m}$ and $40-60 \mathrm{~m}$ in the seven sprints that consisted of either seven regular sprints or three sprints with a sled (sprints 2, 4 and 6) and four sprints without a sled (sprints 1, 3, 5 and 7).

* indicates a significant difference $(p<0.05)$ beween these two sprint times and all those right of the arrow (dashed lines for the resisted runs, black lines for the regular sprints). $\dagger$ indicates a significant difference $(p<0.05)$ beween these two sprint times. 

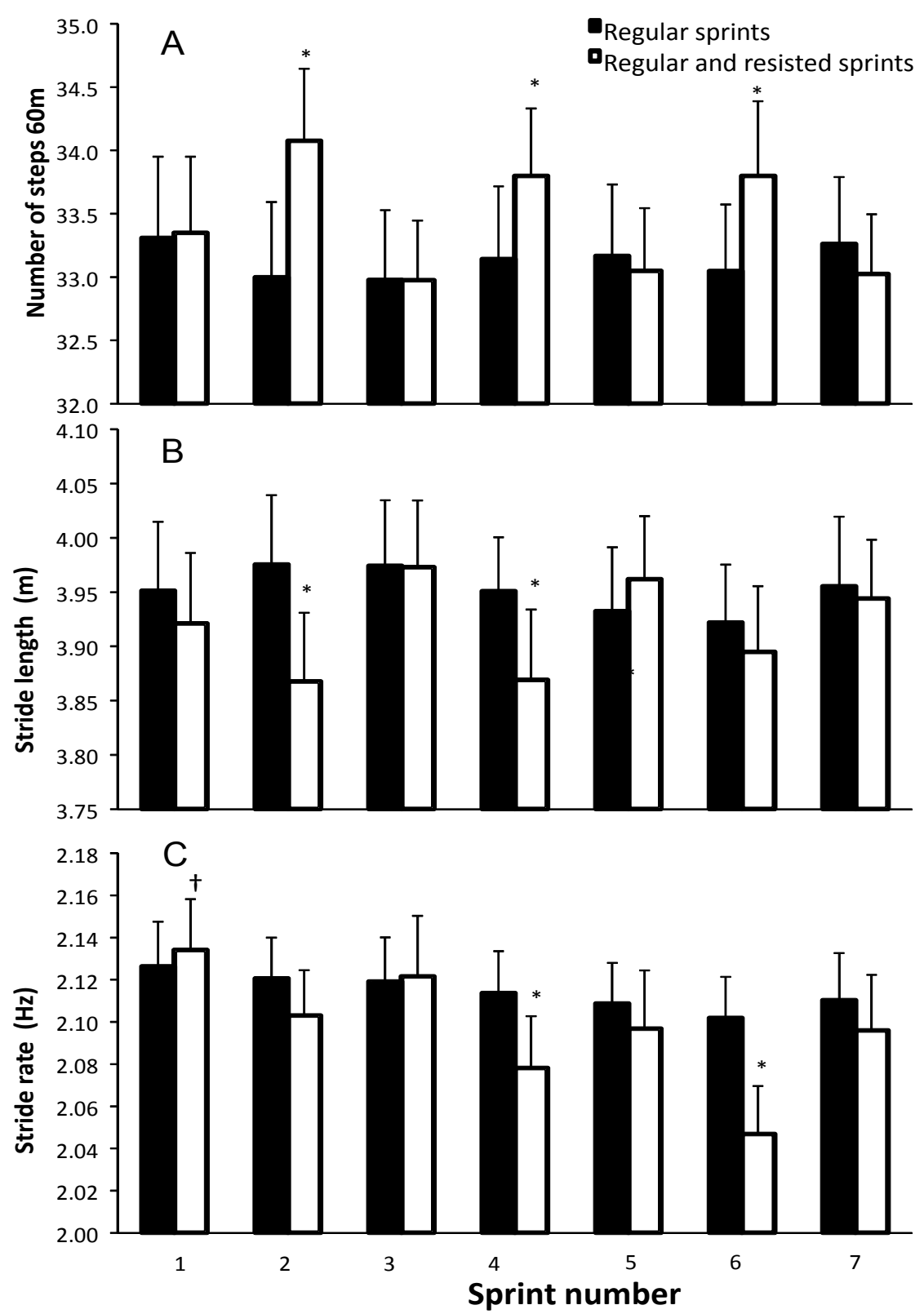

Figure 5 A) Total number of steps ( \pm SD) for the $60 \mathrm{~m}$ sprint B) Stride length C) Stride rate for each regular sprint and sled condition.

* indicates a significant difference $(p<0.05)$ between this sprint with a sled and all regular sprints without a sled.

† indicates a significantly higher stride rate $(p<0.05)$ in this sprint compared to all other sprints except sprint 3 for this condition. 
The seven runs did not affect the step length $\left(\mathrm{F}=0.79 ; \mathrm{p}=0.58 ; \eta^{2}=0.02\right.$; Figure $5 B)$ and step rate $\left(F=1.5 ; p=0.16 ; \eta^{2}=0.04 ;\right.$ Figure $\left.5 C\right)$ in the regular sprints, but in the sled condition step length $\left(F=7.9 ; p<0.001 ; \eta^{2}=0.19\right.$; Figure $\left.5 B\right)$ and rate $\left(F=7.1 ; p<0.001 ; \eta^{2}=0.18\right.$; Figure $\left.5 C\right)$ changed significantly. Post hoc comparison showed that a significantly higher total number of steps were taken, while stride length was significantly lower when pulling a sled compared with regular sprint runs (Figure 5A; Figure 5B). Stride rate followed another pattern; in the sled condition, it decreased after the first sprint (except sprint 3) until sprint 6 (Figure 5C).

\section{DISCUSSION}

The aim of this study was to investigate the acute effect of resisted sprints upon running performances in sprint. The main findings showed no differences in acute effect of resisted sprint running compared to regular sprinting. In addition, it was found that resisted sprints increased the number of steps and decreased stride length. Furthermore, stride rate decreases during the resisted sprint protocol, while this was not found in the regular sprint protocol.

No positive effect (PAP) was found after none of the alternating resisted sprints, which was not in line with the earlier findings [25, 28]. Van den Tillaar and Von Heimburg [25] found in handball players after one resisted $20 \mathrm{~m}$ sprint a positive effect on a $20 \mathrm{~m}$ sprint straight afterwards, while using the same protocol as in the present study (except of using shorter sprints: $20 \mathrm{~m}$ vs. $60 \mathrm{~m}$ ). Handball players sprint only $20 \mathrm{~m}$ regularly in competition, while sprinters train for longer distances (100-200 m). Therefore, it was not expected that there was no enhancement after the first resisted sprint. Perhaps the resistance of the sled was too low to get a positive effect (PAP) on the following $60 \mathrm{~m}$ sprint, because the resistance was only $10 \%$ of body mass. According to a review of Petrakos et al. [16] on training interventions with sled running this weight was not high enough to gain an effect on regular sprinting. According to them, it should be at least $12.5 \%$ of body mass or more to enhance sprint performance after several weeks of training.

However, in the present study the sprint times after 5 min rest at 10, 20, 40 and $60 \mathrm{~m}$ no enhancement was found after the resisted sprint. Furthermore, that from sprint 3 to 4 the times increased (Figures 2-4). These differences in sprint times, mainly caused by the decrease in stride rate (Figure 5C), were also found by Whelan et al. [26]. The possible PAP effect was not found in the $60 \mathrm{~m}$ distance sprints, probably due to fatigue. The $60 \mathrm{~m}$ distance could 
have led to some fatigue in the subjects, therefore not allowing the effect of previous sled running exercises to be highlighted. Possible fatigue was shown by the longer sprint times in sprint five (Figure 2), especially in the first $10 \mathrm{~m}$, and from $20 \mathrm{~m}$ to $60 \mathrm{~m}$ during the regular sprints (Figure 3 and 4). In addition, fatigue was also shown by the slower sprint times from the first resisted sprint to the next one (Figure 2 ) and then again especially from $20 \mathrm{~m}$ to $60 \mathrm{~m}$ (Figure 4). The changes in stride rate could be in line with this assumption, as shown previously $[25,27]$.

The times for the resisted $60 \mathrm{~m}$ sprints were on average over $4.45 \%$ $(0.38 \mathrm{~s})$ slower than for the regular sprints; this is a much lower finding $(-7.5 \%)$ than in the study of Van den Tillaar and Von Heimburg [25]. However, they only conducted $20 \mathrm{~m}$ sprints. Due to the resistance from the sled and limited force subjects can exhibit in their strides it resulted in slower running times. These slower times were mainly caused by the shorter stride length (Figure 5B) and decrease stride rate (Figure 5C), which resulted in increased number of steps over $60 \mathrm{~m}$ sprints. This was also found in the study of Van den Tillaar and Von Heimburg [25]. They showed that also a decreased step length and showed that the lower step frequency was caused by the longer contact times and thereby a lower vertical stiffness.

Zafeiridis et al [29] and Harrison and Bourke [9] observed in their training studies significant improvements in resisted sprints at the acceleration phase $(0-5 \mathrm{~m}, 0-10 \mathrm{~m}$ and $0-20 \mathrm{~m})$, which suggests that resisted sprint training could help at this phase rather than for longer distances. Therefore, in future studies the runs should be reduced to $20-30 \mathrm{~m}$ to avoid fatigue and highlight the possible effect of PAP. However, the weight being pulled is very important. Whelan et al [26] found that pulling an extra $20-30 \%$ of body mass caused limited enhancement, which was unsystematic in nature. Furthermore, there is little clear evidence of fatigue being followed by potentiation. Smith et al [19] suggested a potential for heavier sled resistance to affect improvements, but they found enhancements of around 2.14, 1.21, 2.11 and $2.24 \%$ while running with $0,10,20$ and $30 \%$ loads respectively to have no significant differences between the different loads. Therefore, based Smith et al [19] their findings it seems that running without an extra load ( $0 \%$ load) would give the same enhancement $(2.14 \%)$ as running with a $30 \%$ of body mass load (2.24\%), which indicates that it does not matter if you run with loads or without loads, since extra loads did not add more improvement after an intervention period than the regular running sessions without extra loads.

Sled towing is often used as a part of complex training $[9,23]$ and athletes anecdotally suggest that they run faster after conducting a run with resistance. 
In fact, this study showed that this is not entirely true as subjects ran at the same or decreased velocity. The main factor for this could be fatigue [7]. Data from previous studies shows that repeated exposure to resisted sprint training could be effective in improving initial acceleration [9], suggesting that analysis could focus on the initial sprint phases. This should also be considered in future research.

In the present study, we did not ask the subjects if they felt they ran faster after the resisted sprint runs, which could have given more detailed information about their awareness [2]. Investigating if awareness is influenced by the level of training experience could also be an interesting perspective to include in future studies. Furthermore, including electromyography (EMG) measurements to investigate if there are differences in muscle activation amplitude, firing frequency and timing that influence the stride rate, together with more detailed kinematics (joint angles) should be performed to get a better understanding of what changes are due to sled running and to show if PAP and/or fatigue occurs during these types of training.

Our study showed that there were no acute PAP effects of resisted sprints upon running performances in sprints. These results have important implications for athletes, coaches and physical-training staff. They show that anecdotal evidence suggested by athletes - that they run faster after conducting a run with resistance - is not correct. Based on the results of our study we do not advise resisted sprints on this level of athletes to be used with long distances $(60 \mathrm{~m})$ with the goal of a PAP effect, since this does not occur or fatigue is higher. However, resisted runs could be included in regular sprint training as more work is allowed, without producing more fatigue than seven regular $60 \mathrm{~m}$ runs (Figure 4). Perhaps implementing resisted runs over shorter distances, higher level performing athletes or more strength-trained individuals could be effective in improving initial acceleration because of the PAP effect; this should be considered in future research.

\section{REFERENCES}

1. Alcaraz PE, Palao JM, Elvira JL, Linthorne NP. (2008) Effects of three types of resisted sprint training devices on the kinematics of sprinting at maximum velocity. J Strength Cond Res, 22: 890-897

2. Barnes KR, Hopkins WG, McGuigan MR, Kilding AE. (2015) Warm-up with a weighted vest improves running performance via leg stiffness and running economy. J Sci Med Sport, 18: 103-108

3. Bishop D. (2003) Warm Up I: Potential mechanisms and the effects of passive warm up on exercise performance. Sports Med, 33: 439-454 
4. Clark KP, Stearne DJ, Walts CT, Miller AD. (2010) The longitudinal effects of resisted sprint training using weighted sleds vs. weighted vests. J Strength Cond Res, 24: 3287-3295

5. Cohen J. (1988) Statistical Power Analysis for the Behavioral Sciences. Hillsdale, NJ, England: Lawrence Erlbaum Associates

6. Corn RJ, Knudson D. (2003) Effect of elastic-cord towing on the kinematics of the acceleration phase of sprinting. J Strength Cond Res, 17: 72-75

7. Goodall S, Charlton K, Howatson G, Thomas K. (2015) Neuromuscular fatigability during repeated-sprint exercise in male athletes. Med Sci Sports Exerc, 47: $528-536$

8. Hamada T, Sale DG, MacDougall JD, Tarnopolsky MA. (2000) Postactivation potentiation, fiber type, and twitch contraction time in human knee extensor muscles. J Appl Physiol, 88: 2131-2137

9. Harrison AJ, Bourke G. (2009) The effect of resisted sprint training on speed and strength performancein male rugby players. J Strength Cond Res, 23: 275283

10. Hrysomallis C. (2012) The effectiveness of resisted movement training on sprinting and jumping performance. J Strength Cond Res, 26: 299-306

11. Konstantinos Z, Athanasia S, Polyxeni A, Georgios P, Elias Z, Charilaos T. (2014) Acute effects of loading using a weighted vest on running performance. Biol Exerc, 10: 53-65

12. Kristensen GO, van den Tillaar R, Ettema GJ. (2006) Velocity specificity in early-phase sprint training. J Strength Cond Res, 20: 833-837

13. Lockie RG, Murphy AJ, Jeffriess MD, Callaghan SJ. (2013) Step kinematic predictors of short sprint performance in field sport athletes. Serb J Sports Sci, 7: $71-77$

14. Makaruk B, Sozanski H, Makaruk H, Sacewicz T. (2013) The effects of resisted sprint training on speed performance in women. Human Mov, 14: 116-122

15. Paulson S, Braun WA. (2011) The influence of parachute-resisted sprinting on running mechanics in collegiate track athletes. J Strength Cond Res, 25: 16801685

16. Petrakos G, Morin JB, Egan B. (2016) Resisted sled sprint training to improve sprint performance: A systematic review. Sports Med, 46: 381-400

17. Reiman MP, Peintner AM, Boehner AL, Cameron CN, Murphy JR, Carter JW. (2010) Effects of dynamic warm-up with and without a weighted vest on lower extremity power performance of high school male athletes. J Strength Cond Res, 24: 3387-3395

18. Robbins DW. (2005) Postactivation potentiation and its practical applicability: A brief review. J Strength Cond Res, 19: 453-458

19. Smith CE, Hannon JC, McGladrey B, Shultz B, Eisenman P, Lyons B. (2014) The effects of a postactivation potentiation warm-up on subsequent sprint performance. Hum Mov, 15: 33-41 
20. Spinks CD, Murphy AJ, Spinks WL, Lockie RG. (2007) The effects of resisted sprint training on acceleration performance and kinematics in soccer, rugby union and Australian football players. J Strength Cond Res, 21: 77-85

21. Tillin NA, Bishop D. (2009) Factors modulating post-activation potentiation and its effect on performance of subsequent explosive activities. Sports Med, 39: $147-166$

22. Tsimachidis C, Patikas D, Galazoulas C, Bassa E, Kotzamanidis C. (2013) The post-activation potentiation effect on sprint performance after combined resistance/sprint training in junior basketball players. J Sports Sci, 31: 1117-1124

23. Upton DE. (2012) The effect of assisted and resisted sprint training on acceleration and velocity in division IA female soccer athletes. J Strength Cond Res, 25: 2645-2652

24. van den Tillaar R. (2004) Effect of different training programs on the velocity of overarm throwing: a brief review. J Strength Cond Res, 18: 388-396

25. van den Tillaar R, Von Heimburg E. (2017) Comparison of different sprint training sessions with assisted and resisted running: effects on performance and kinematics in $20 \mathrm{~m}$ sprints. . Human Movement, 18:

26. Whelan N, O’Regan C, Harrison AJ. (2014) Resisted sprints do not actually enhance sprinting performance. J Strength Cond Res, 28: 1858-1866

27. Williams KR, Snow R, Agruss C. (1991) Changes in distance running kinematics with fatigue. Int J Sport Biomech, 7: 138-162

28. Wyland TP, Van Dorin JD, Reyes GF. (2015) Postactivation potentation effects from accommodating resistance combined with heavy back squats on short sprint performance. J Strength Cond Res, 29: 3115-3123

29. Zafeiridis A, Saraslanidis P, Manou V, Ioakimidis P, Dipla K, Kellis S. (2005) The effects of resisted sled-pulling sprint training on acceleration and maximum speed performance. J Sports Med Phys Fit, 45: 284-290

\section{Correspondence to:}

Prof. Roland van den Tillaar PhD.

Department of Sports Sciences and Physical Education

Nord University

Odins veg 23

7603 Levanger, Norway

Phone: +47 57671883

Fax: +4774112001

E-mail: roland.v.tillaar@nord.no 